Eur. J. Clin. Chem. Clin. Biochem.

Vol. 31, 1993, pp. 743-747

(C) 1993 Walter de Gruyter \& Co.

Berlin · New York

\title{
Apolipoprotein E to B Ratio: A Marker for Type III Hyperlipoproteinaemia
}

By W. März ${ }^{1}$, G. Feussner ${ }^{2}$, R. Siekmeier ${ }^{1}$, Bettina Donnerhak ${ }^{1}$, L. Schaaf ${ }^{3}$, V. Ruzicka ${ }^{1}$ and W. Groß ${ }^{1}$

${ }^{1}$ Gustav Embden-Zentrum der Biologischen Chemie, J. W. Goethe-Universität, Frankfurt am Main, Germany

${ }^{2}$ Abteilung Innere Medizin I (Endokrinologie und Stoffwechsel), Medizinische Klinik der Universität Heidelberg, Heidelberg, Germany

${ }^{3}$ Zentrum der Inneren Medizin, Abteilung für Endokrinologie, J. W. Goethe-Universität, Frankfurt am Main, Germany

(Received November 3, 1992/August 10, 1993)

Summary: Apolipoproteins B and E were determined in 40 patients with type III hyperlipoproteinaemia (familial dysbetalipoproteinaemia) and in 48 patients with other types of hyperlipoproteinaemia matched for cholesterol and triacylglycerols. In type III patients, apolipoprotein E was increased and apolipoprotein B was lower than in other types of hyperlipoproteinaemia. The apolipoprotein $\mathrm{E}$ to apolipoprotein $\mathrm{B}$ ratio almost completely discriminated between type III and other types of hyperlipoproteinaemia. Assuming a cutoff value of 0.09 for the apolipoprotein $\mathrm{E}$ to apolipoprotein B ratio, diagnostic sensitivity was $95 \%$ and specificity was $88 \%$. It is concluded that the apolipoprotein $\mathrm{E}$ to apolipoprotein $\mathrm{B}$ ratio represents the firstline screening quantity of choice for the identification of patients with type III hyperlipoproteinaemia in the clinical laboratory.

\section{Introduction}

Familial type III hyperlipoproteinaemia is characterized by the accumulation of cholesterol-rich remnants derived from the partial catabolism of chylomicrons and very low density lipoproteins. Type III patients are at high risk of developing premature coronary and peripheral atherosclerosis (1).

Pathogenetically, type III hyperlipoproțeinaemia is related to dysfunctional isoforms of apolipoprotein E, a $M_{\mathrm{r}} 34000$ glycoprotein normally associated with very low density and high density lipoproteins $(1-4)$. More than $90 \%$ of type III patients are homozygous for the apolipoprotein E2 isoform which does not bind normally to apolipoprotein B,E-receptors (5-7), and this accounts for the impaired clearance of remnants in type III hyperlipoproteinaemia. However, the apolipoprotein E2/2 phenotype is much more frequent than type III hyperlipoproteinaemia. Only one in 20 apolipoprotein E2/2 homozygotes will actually develop type III hyperlipoproteinaemia, the remaining ones being normolipidaemic or even hypolipidaemic. This has led to the suggestion that further genetic, metabolic, or environmental factors are required to express type III hyperlipoproteinaemia $(1,8-11)$.

There is no simple test to establish the biochemical diagnosis of type III hyperlipoproteinaemia. Currently, the following criteria are applied: cholesterol and triacylglycerols are elevated to about the same extent, cutaneous xanthomas are present, lipoprotein . electrophoresis reveals a broad $\beta$-band, the VLDLcholesterol to plasma triacylglycerol $[\mathrm{mmol} / \mathrm{l}]$ ratio exceeds $0.68, \beta$-migrating particles are found in the VLDL fraction, and the apolipoprotein $\mathrm{E}$ phenotype is $2 / 2$.

However, none of these criteria is compelling. Cholesterol and triacylglycerols may vary widely in and among type III subjects. Cutaneous xanthomas occur in only about $50 \%$ of the patients, and the demonstration of a broad $\beta$-band in agarose gel electrophoresis of whole plasma is often misleading. Obviously, 
the most reliable markers for type III hyperlipoproteinaemia are an increased VLDL-cholesterol to plasma triacylglycerol ratio, the demonstration of $\beta$ VLDL, and apolipoprotein E2/2 homozygosity. ${ }^{1}$ ) This means that laboratory diagnosis of type III hyperlipoproteinaemia depends on sophisticated methods such as ultracentrifugation and apolipoprotein $E$ phenotyping which are usually available to specialized laboratories only.

On the other hand, there are several points calling for a simple way to recognize type III hyperlipoproteinaemia in the clinical setting. Firstly, affected patients carry a tremendous risk of developing coronary artery disease and peripheral vascular disease. Secondly, the diagnosis of type III hyperlipoproteinaemia has a considerable therapeutic impact. Type III patients usually respond well to the elimination of disease promoting factors and/or to dietary treatment, and once lipoprotein metabolism has been normalized, former type III patients may even be protected from premature atherosclerosis due to the low concentration of LDL found in normolipidaemic E2 homozygotes (1).

It has been known for a long time that apolipoprotein E concentrations are increased in type III hyperlipoproteinaemia (12), whereas apolipoprotein B is normal or low. Nowdays, both apolipoprotein $E$ and apolipoprotein B can easily be quantified by automated methods. We have therefore studied the relevance of apolipoprotein $\mathrm{E}$ and apolipoprotein $\mathrm{B}$ determinations to the differential diagnosis of type III hyperlipoproteinaemia.

\section{Materials and Methods}

Blood sampling

Fasting blood samples were drawn into tubes containing EDTA - $\mathrm{K}_{2}$ at a final concentration of $1.5-2.0 \mathrm{~g} / \mathrm{l}$ (Sarstedt, Nümbrecht, Germany). Plasma was recovered by centrifugation and stored at $4{ }^{\circ} \mathrm{C}$.

\section{Lipoprotein and apolipoprotein analysis}

Cholesterol (Monotest, CHOD-PAP) and triacylglycerols (GPO-PAP) were measured using reagents from Boehringer Mannheim (Germany). VLDL-cholesterol was determined by preparative ultracentrifugation at $\mathrm{d}_{20}{ }^{\circ} \mathrm{C}=1.0063 \mathrm{~kg} / \mathrm{l}(2.35$ $\cdot 10^{8} \mathrm{~g} \cdot \mathrm{min}$ ) as described (13). High density lipoprotein cholesterol was measured after precipitation of apolipoprotein Bcontaining lipoproteins with sodium phosphotungstic acid and $\mathrm{MgCl}_{2}$ (13). LDL-cholesterol was calculated as the difference between total cholesterol and the sum of VLDL-cholesterol and high density lipoprotein cholesterol (13).

$\left.{ }^{1}\right)$ Homozygosity for apolipoprotein E2 is highly suggestive of type III hyperlipoproteinaemia, but other apolipoprotein $\mathrm{E}$ phenotypes do not rule out the disorder.
Apolipoproteins $\mathrm{B}$ and $\mathrm{E}$ were determined by kinetic nephelometry (Array Protein System, Beckman Diagnostics, Brea, CA). The assay for apolipoprotein B was calibrated with the apolipoprotein calibrator APO CAL from Beckman Diagnostics, which is standardized against the WHO international reference material. The assay for apolipoprotein $\mathrm{E}$ was also calibrated using a standard provided by Beckman. Electrophoresis of lipoproteins was carried out as described by Neubeck et al. (14). Apolipoprotein E phenotyping was performed by isolectric focusing of delipidated VLDL (15) or by immunoblotting (16).

\section{Subjects}

Fourty patients (18 male, 22 female, age range $31-72$ years) with type III hyperlipoproteinaemia recruited at the Department of Internal Medicine in Heidelberg and at the Centre of Internal Medicine in Frankfurt were studied. Among these type III patients, 16 suffered from peripheral vascular disease. Cerebrovascular disease and coronary artery disease were present in 9 and 6 cases, respectively. The diagnosis of type III hyperlipoproteinaemia was made if all the following criteria were met: cholesterol above $5.2 \mathrm{mmol} / \mathrm{l}$ and/or triacylglycerols above $2.3 \mathrm{mmol} / \mathrm{l}$; the ratio of VLDL-cholesterol to plasma triacylglycerols [mmol/l] greater than 0.68; and detection of VLDL particles with $\beta$-mobility. Phenotypically, all type III patients were E2/2 homozygotes. As controls, we studied samples from patients with primary hyperlipoproteinaemia other than type III. Because we wished to simulate the diagnostic situation, we selected control samples that were suggestive of type III hyperlipoproteinaemia on the basis of their cholesterol and triacylglycerol concentrations. In all control samples the ratio of VLDL-cholesterol to total triacylglycerols was lower than 0.68 , and none had the apolipoprotein E2/2 phenotype. Twenty eight controls had type IV hyperlipoproteinaemia, 17 had type IIb and 3 had type IIa hyperlipoproteinaemia; eleven individuals suffered from coronary artery disease, as diagnosed clinically and by an exercise electrocardiogram.

\section{Results}

Table 1 contains mean lipid and apolipoprotein concentrations in the two patient groups. As a result of matching, the groups were similar with respect to cholesterol and triacylglycerols. In spite of this, apolipoprotein B was lower and apolipoprotein E was higher in type III subjects than in non-type III subjects. Consequently, the apolipoprotein $\mathrm{E} / \mathrm{B}$ ratio was different. Figure 1 shows that the apolipoprotein E/B ratio allows almost complete discrimination of the groups. Using a threshold value of 0.09 for the apolipoprotein E/B ratio, type III hyperlipoproteinaemia would be detected with a sensitivity of $95 \%$, the specificity being $88 \%$. If one assumes that the prevalence of type III hyperlipoproteinaemia is 1 in 100 patients with hyperlipidaemia, the predictive value of the positive test (apolipoprotein E/B > 0.09) is 0.07 , the predictive value of the negative test is 1.00 , and diagnostic efficacy is 0.88 .

In plasma, a considerable fraction of apolipoprotein $\mathrm{E}$ is associated with triacylglycerol-rich lipoproteins. Therefore, increases in triacyćlglycerols are generally attended by increases in apolipoprotein E. In fact, in 
Tab. 1. Lipids [mmol/l] and apolipoproteins in patients with type III hyperlipoproteinacmia and in patients with other types of hyperlipoproteinaemia.

\begin{tabular}{llc}
\hline & $\begin{array}{l}\text { Type III patients } \\
n=40\end{array}$ & $\begin{array}{l}\text { Controls } \\
n=48\end{array}$ \\
\hline Cholesterol & $7.43 \pm 2.69^{\mathrm{a}}$ & $7.38 \pm 1.58$ \\
& $6.86(4.04-18.31)^{\mathrm{b}}$ & $7.25(4.56-12.64)$ \\
Triacylglycerols & $4.15 \pm 2.67$ & $4.98 \pm 2.63$ \\
& $3.15(1.19-11.10)$ & $4.69(0.92-11.76)$ \\
HDL-cholesterol & $1.01 \pm 0.21$ & $0.96 \pm 0.26$ \\
& $1.01(0.41-1.37)$ & $0.88(0.54-1.79)$ \\
Apolipoprotcin B [g/l] & $0.91 \pm 0.27$ & $1.64 \pm 0.56$ \\
& $0.83(0.52-2.04)$ & $1.72(0.88-3.01)$ \\
Apolipoprotcin E [mg/l] & $174 \pm 70$ & $88 \pm 65$ \\
Ratio apolipoprotein E/apolipoprotein B & $157(71-390)$ & $74(10-389)$ \\
& $0.197 \pm 0.073$ & $0.056 \pm 0.037$ \\
\end{tabular}

- means \pm standard deviation

medians and ranges (2.5 $5^{\text {th }}$ and $97.5^{\text {th }}$ percentiles)

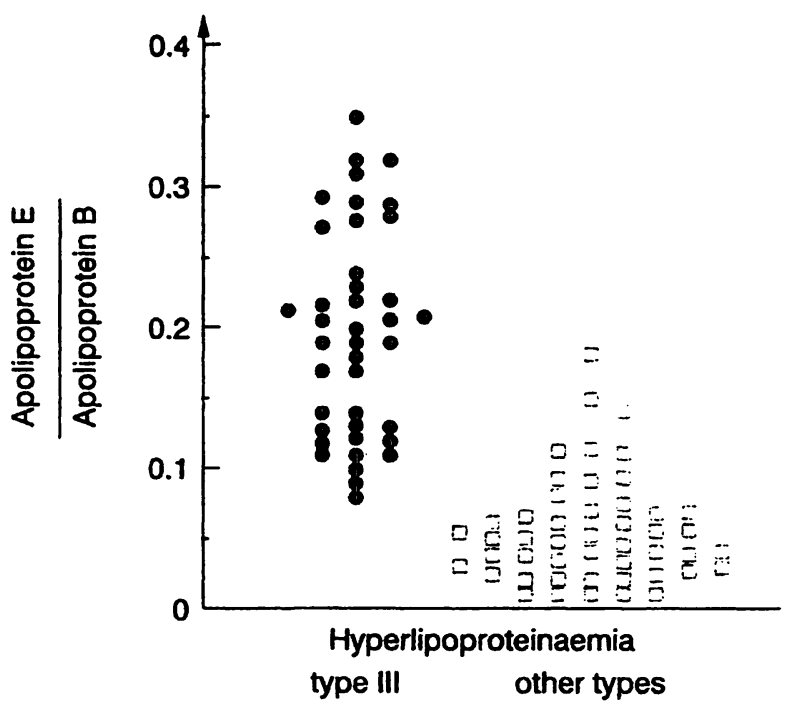

Fig. 1. Apolipoprotein E to apolipoprotein B ratio in 40 patients with type III hyperlipoproteinaemia (closed circles) and in 48 patients with other types of hyperlipoproteinaemia (open squares), matched for cholesterol and triacylglycerols.

type III patients as well as in the controls, the apolipoprotein $E / B$ ratio was positively correlated with triacylglycerols, the correlation coefficients being 0.483 and 0.632 , respectively. However, as shown in figure 2, at any given triacylglycerol concentration the apolipoprotein E/B ratio was higher in type III patients than in the control group. Figure 2 also illustrates that if the apolipoprotein E/B cut-off is set in favour of maximal sensitivity, e.g. at 0.07 , triacylglycerol concentrations may be disregarded. In contrast, if specificity is to be increased, the apolipoprotein $\mathrm{E} / \mathrm{B}$ cut-off has to be adjusted for triacyclglycerols, tentative threshold values being 0.09 in samples with triacylglycerols below $5.0 \mathrm{~g} / \mathrm{l}$, and 0.2 in samples with higher triacylglycerols.

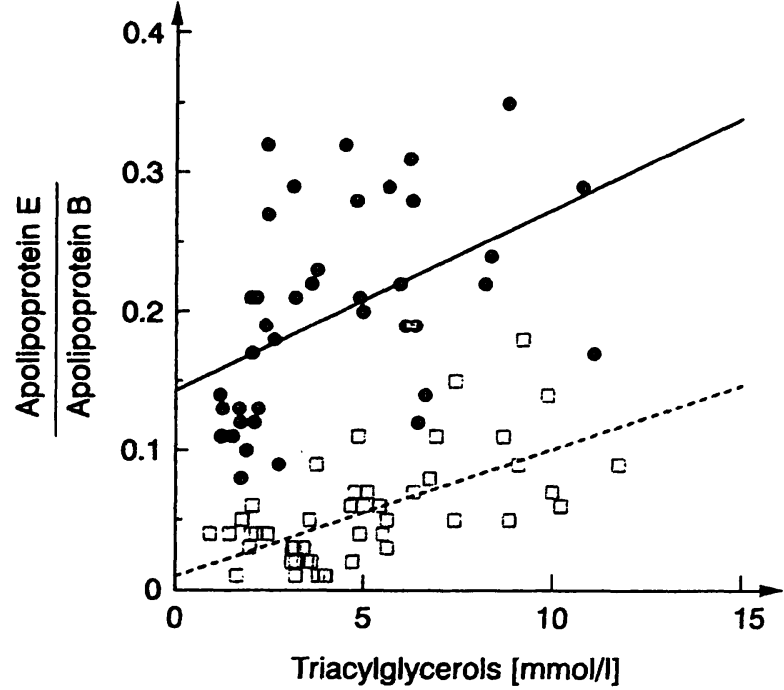

Fig. 2. Apolipoprotein E to apolipoprotein B ratio in 40 patients with type III hyperlipoproteinaemia (closed circles, solid line) and in 48 patients with other types of hyperlipoproteinaemia (open squares, dashed line) plotted versus plasma triacylglycerols.

Equations of the regression lines are as follows:

a) type III hyperlipoproteinaemia (solid line): apolipoprotein $\mathrm{E} / \mathrm{B}=0.013 \cdot$ triacylglycerols +0.14 $r=0.483$;

b) other types of hyperlipoproteinaemia (dashed line): apolipoprotein $\mathrm{E} / \mathrm{B}=0.009 \cdot$ triacylglycerols +0.009 ; $r=0.632$.

\section{Discussion}

Our data show that apolipoprotein E is markedly increased in type III hyperlipoproteinaemia, while apolipoprotein B is low or normal. This constellation is not only seen in type III hyperlipoproteinaemia, but also in normolipidaemic E2/2 homozygotes, and it relates to the well-documented functional abnormality of apolipoprotein E2 (5-7). 
Apart from several rare variants $(17-23)$, there are three common alleles at the apolipoprotein $\mathrm{E}$ gene locus ( $\varepsilon 2, \varepsilon 3$, and $\varepsilon 4)$, encoding for the isoforms $E 2$, E3, and E4 (24). More than $90 \%$ of patients with type III hyperlipoproteinaemia are of the E2/2 phenotype (9). In vitro, apolipoprotein E2 is defective in binding to apolipoprotein B,E-receptors (5-7). Since the clearance of both chylomicron and VLDL remnants depends on apolipoprotein E, even normolipidaemic apolipoprotein E2/2 homozygotes accumulate small amounts of remnant particles in the circulation (dysbetalipoproteinaemia). The flux of dietary cholesterol into the liver is decreased and hepatic apolipoprotein B,E-receptors are up-regulated. Therefore, E2 homozygotes have low LDL-cholesterol and apolipoprotein B concentrations. In contrast, the presence of at least one apolipoprotein E4 allele is associated with high levels of LDL-cholesterol and apolipoprotein $B(1,9-11)$.

In type III hyperlipoproteinaemia, remnant accumulation is largely accentuated by the presence of at least one factor other than apolipoprotein E2 homozygosity, exogenous or genetic. In general, the remnant particles found in type III hyperlipoproteinaemia impose a very high risk of atherosclerosis. $\beta$-VLDL from subjects with type III hyperlipoproteinaemia are avidly taken up by macrophages, which are thereby converted into lipid-storing foam cells, one of the key cell types of atherosclerotic lesions (25). Since type III hyperlipoproteinaemia responds well to the elimination of disease-promoting conditions (1) and to dietary treatment (26), its recognition is of vital importance for the affected individuals.

Unfortunately, there is hardly any simple and straightforward screening method for type III hyperlipoproteinaemia in the clinical laboratory. Thus, many patients with this disorder have so far remained undiagnosed.

Generally speaking, samples in which both cholesterol and triacylglycerols are about two- to fourfold the normal value are suggestive of type III hyperlipoproteinaemia. However, in routine practice, methods such as ultracentrifugation or apolipoprotein $\mathrm{E}$ phenotyping are not available for confirming or rejecting the diagnosis of type III hyperlipoproteinaemia. There- fore, a simple, yet sensitive test is required to identify patients with type III hyperlipoproteinaemia. In the present study, we show that the apolipoprotein E/ apolipoprotein B ratio almost completely discriminates between type III hyperlipoproteinaemia and other types of hyperlipoproteinaemia with approximately the same concentrations of total cholesterol, triacylglycerols and high density lipoprotein cholesterol. Using a cut-off point of 0.09 for the apolipoprotein $\mathrm{E} / \mathrm{B}$ ratio, sensitivity and specificity would be $95 \%$ and $88 \%$, respectively. Assuming that the prevalence of type III hyperlipoproteinaemia is 1 in 100 patients with hyperlipidaemia, the predictive value of the positive test (apolipoprotein E/B $>0.09$ ) would be 0.07 , the predictive value of the negative test would be 1.00 and diagnostic efficacy would be 0.88 . These estimates are very close to the characteristics of tests applied to the screening for rare inborn errors of metabolism such as phenylketonuria, cystic fibrosis, hypothyroidism or muscular dystrophies (27).

Since automated assays for both apolipoprotein B and apolipoprotein $\mathrm{E}$ have recently become available, these determinations can conveniently be performed in any clinical laboratory. The values for the apolipoprotein $\mathrm{E}$ to apolipoprotein $\mathrm{B}$ ratio obtained in this work do, of course, depend on the immunoassay method used. Our apolipoprotein B immunoassay was calibrated against the WHO international reference material. At present, no such material is available for apolipoprotein E. Therefore, the cut-off values derived here may need adjustment in the future. In the meantime, laboratories using our approach to the screening for type III hyperlipoproteinaemia will have to establish own normal ranges.

The apolipoprotein $\mathrm{E}$ to apolipoprotein $\mathrm{B}$ ratio does not replace the classical means of confirming type III hyperlipoproteinaemia, but it may prove suitable as a first line screening parameter for identifying patients in need of additional testing with technically demanding procedures such as ultracentrifugation or apolipoprotein E phenotyping.

\section{Acknowledgement}

The authors thank Mrs. Sabine Cezanne and Mrs. Daniela Wittmann for their skilful technical assistance. 


\section{References}

1. Mahley, R. W. \& Rall, S. C. (1989) Type III hyperlipoproteinaemia. In: The Metabolic Basis of Inherited Disease, $6^{\text {th }}$ edn. Vol. I. (Scriver, C. R., Beaudet, A. L., Sly, W. S. \& Valle, D., eds.) pp. 1195-1213, McGraw Hill Book Company, New York.

2. Rall, S. C., Weisgraber, K. H. \& Mahley, R. W. (1982) Human apolipoprotein $\mathrm{E}$. The complete amino acid sequence. J. Biol. Chem. 257, 4171-4178.

3. Paik, Y. K., Chang, D. J., Reardon, C. A., Davies, G. E., Mahley, R. W. \& Taylor, J. M. (1985) Nucleotide sequence and structure of the human apolipoprotein $\mathrm{E}$ gene. Proc. Natl. Acad. Sci. U. S. A. 82, 3445-3449.

4. Mahley, R. W. (1988) Apolipoprotein E: Cholesterol transport protein with expanding role in cell biology. Science $240,622-630$

5. Schneider, W. J., Kovanen, P. T., Brown, M. S., Goldstein, J. L., Utermann, G., Weber, W., Havel, R. J., Kotite, L., Kane, J. P., Innerarity, T. L. \& Mahley, R. W. (1981) Familial dysbetalipoproteinemia: Abnormal binding of mutant apolipoprotein $E$ to low density lipoprotein receptors of human fibroblasts and membranes from liver and adrenals of rats, rabbits and cows. J. Clin. Invest. 68, 1075-1085.

6. Lalazar, A., Weisgraber, K. H., Rall, S. C., Giladi, H., Innerarity, T. L., Levanon, A. Z., Boyles, J. K., Amit, B., Gorecki, M., Mahley, R. W. \& Vogel, T. (1988) Site-specific mutagenesis of human apolipoprotein $\mathrm{E}$. Receptor binding activity of variants with single amino acid substitutions. J. Biol. Chem. 263, 3542-3545.

7. Weisgraber, K. H., Innerarity, T. L. \& Mahley, R. W. (1982) Abnormal lipoprotein receptor-binding activity of the human apo $E$ apoprotein due to cysteine-arginine interchange at a single site. J. Biol. Chem. 257, 2518-2521.

8. Utermann, G., Hees, M. \& Steinmetz, A. (1977) Polymorphism of apolipoprotein $\mathrm{E}$ and occurrence of dysbetalipoproteinaemia in man. Nature 269, 604-607.

9. Utermann, G. (1987) Apolipoprotein E polymorphism in health and disease. Am. Heart. J. 113, 433-440.

10. Gregg, R. E. \& Brewer, H. B. jr. (1988) The role of apolipoprotein $\mathrm{E}$ and lipoprotein receptors in modulating the in vivo metabolism of apolipoprotein B-containing lipoproteins in human plasma. Clin. Chem. 34, B28-B32.

11. Davignon, J., Gregg, R. E. \& Sing, C. F. (1988) Apolipoprotein $\mathrm{E}$ polymorphism and atherosclerosis. Arteriosclerosis $8,1-21$.

12. Havel, R. J. \& Kane, J. P. (1972) Primary dysbetalipoproteinemia: Predominance of a specific apoprotein species in triglyceride rich lipoproteins. Proc. Natl. Acad. Sci. U. S. A. 70, 2015-2019.

13. März, W. \& Groß, W. (1986) Analysis of plasma lipoproteins by ultracentrifugation in a new fixed angle rotor: Evaluation of a phosphotungstic acid/ $\mathrm{MgCl}_{2}$ precipitation and a quantitative lipoprotein electrophoresis assay. Clin. Chim. Acta $160,1-18$.

14. Neubeck, W., Wieland, H., Habenicht, A., Müller, P., Baggio, G. \& Seidel, D. (1977) Improved assessment of plasma lipoprotein patterns. III. Direct measurement of lipoproteins after gel-electrophoresis. Clin. Chem. 23, 1296-1300.
15. Warnick, G. R., Albers, J. J. \& Hazzard, W. R. (1979) Gel isoelectric focusing method for specific diagnosis of familial hyperlipoproteinaemia type 3. Clin. Chem. 25, 179-284.

16. März, W., Cezanne, S. \& Groß, W. (1991) Phenotyping of apolipoprotein $\mathrm{E}$ by immunoblotting in immobilized $\mathrm{pH}$ gradients. Electrophoresis 12, 59-63.

17. Rall, S. C., Weisgraber, K. H., Innerarity, T. L., Bersot, T. P. \& Mahley, R. W. (1983) Identification of a new structural variant of human apolipoprotein E, E2 (Lys ${ }_{146} \rightarrow$ Gln), in a type III hyperlipoproteinaemia subject with the E3/2 phenotype. J. Clin. Invest. 72, 1288-1297.

18. Weisgraber, K. H., Rall, S. C., Innerarity, T. L., Mahley, R. W., Kuusi, T. \& Ehnholm, C. (1984) A novel electrophoretic variant of human apolipoprotein E. Identification and characterization of apolipoprotein E1. J. Clin. Invest. 73, 1024-1033.

19. Yamamura, T., Yamamato, A., Hiramori, K. \& Nambu, S. (1984) A new isoform of apolipoprotein E - apo E-5 associated with hyperlipidaemia and atherosclerosis. Atherosclerosis $50,159-172$.

20. Ghiselli, G. V., Gregg, R. E. \& Brewer, H. B. jr. (1984) Apolipoprotein E-Bethesda. Isolation and partial characterization of a variant of human apolipoprotein $\mathrm{E}$ isolated from very low density lipoproteins. Biochim. Biophys. Acta 794, 333-339.

21. Wardell, M. R., Weisgraber, K. H., Havekes, L. M. \& Rall, S. C. (1989) Apolipoprotein E3-Leiden contains a sevenamino acid insertion that is a tandem repeat of residues 121-127. J. Biol. Chem. 264, 21205-21210.

22. Wardell, M. R., Brennan, S. O., Janus, E. D., Fraser, R. \& Carrell, R. W. (1987) Apolipoprotein E2-Christchurch (136 $\mathrm{Arg} \rightarrow$ Ser). A new variant of human apolipoprotein $\mathrm{E}$ in a patient with type III hyperlipoproteinaemia. J. Clin. Invest. $80,483-490$.

23. Tajima, S., Yamamura, T., Menju, M. \& Yamamoto, A. (1989) Analysis of apolipoprotein E7 (apolipoprotein ESuita) gene from a patient with hyperlipoproteinemia. J. Biochem. 105, 249-253.

24. Weisgraber, K. H., Rall, S. C. \& Mahley, R. W. (1981) Human apoprotein $\mathrm{E}$ heterogeneity. Cysteine arginine interchanges in the amino acid sequence of the apo- $E$ isoforms. J. Biol. Chem. 256, 9077-9083.

25. Brown, M. S. \& Goldstein, J. L. (1983) Cholesterol metabolism in the macrophage: Implications for cholesterol deposition in atherosclerosis. Ann. Rev. Biochem. 52, 223261.

26. Brewer, H. B. Jr., Zech, L. A., Gregg, R. W., Schwartz, D. \& Schaefer, J. (1983) Type III hyperlipoproteinaemia: Diagnosis, molecular defects, pathology, and treatment. Ann. Int. Med. 98, 623-640.

27. Thomas, L. (1992) Labor und Diagnose. Indikation und Bewertung von Laborbefunden für die medizinische Diagnostik, 4. Aufl., pp. 1421-1441, Medizinische Verlagsgesellschaft, Marburg.

\section{Dr. Winfried März}

Gustav Embden-Center of Biological Chemistry

University Hospital

J. W. Goethe-University

Theodor-Stern-Kai 7

D-60590 Frankfurt/Main

Germany 
